\title{
Overlap of Viviparous1 (VP1) and Abscisic Acid Response Elements in the Em Promoter: G-Box Elements Are Sufficient but Not Necessary for VP1 Transactivation
}

\author{
Vimla Vasil,, ${ }^{a}$ William R. Marcotte, Jr., ${ }^{\text {b,1 }}$ Leonard Rosenkrans, ${ }^{a}$ Suzy M. Cocciolone, ${ }^{a}$ Indra K. Vasil, ${ }^{a}$ \\ Ralph S. Quatrano, ${ }^{\mathrm{C}}$ and Donald R. McCarty ${ }^{\mathrm{a}, 2}$ \\ a Program in Plant Molecular and Cellular Biology, Horticultural Sciences Department, 2237 Fifield Hall, Institute of Food \\ and Agricultural Sciences, University of Florida, Gainesville, Florida 32611 \\ b Department of Biological Sciences, 132 Long Hall, Clemson University, Clemson, South Carolina 29634 \\ c Biology Department, University of North Carolina, Chapel Hill, North Carolina 27599
}

The relationship between promoter sequencs that mediate Viviparous1 (VP1) transactivation and regulation by abscisic acid (ABA) in the wheat Em promoter was investigated using deletion analysis and directed mutagenesis. The Em1a G-box is strongly coupled to VP1 transactivation as well as to ABA regulation; however, the Em promoter includes additional components that can support VP1 transactivation without ABA responsiveness or synergism. Oligonucleotide tetramers of several G-box sequences, including Em1a, Em1b, and the dyad G-box element from the UV light-regulated parsley chalcone synthase gene, were sufficient to confer VP1 transactivation and the synergistic interaction with ABA to the -45 cauliflower mosaic virus $35 S$ core promoter. These data suggest that VP1 can activate transcription through at least two classes of cis-acting sequences, including the G-box elements and the Sph regulatory motif found in the C1 promoter. The contrasting roles of these motifs in the $\mathrm{Em}$ and $\mathrm{C} 1$ promoters suggest a basis for the differential regulation of the corresponding genes by VP1.

\section{INTRODUCTION}

Abscisic acid (ABA) is a key regulator of gene expression associated with late embryogenesis in seed plants (Skriver and Mundy, 1990; Rock and Quatrano, 1994). Among the best characterized ABA-regulated genes is the $E m$ gene expressed in developing wheat embryos (Marcotte et al., 1989; Quatrano et al., 1993). Analyses of ABA-responsive sequences in the Em promoter have shown that a complex of G-box elements (complex I), which includes the Em1a motif (CACGTGGC), is necessary for ABA regulation (Marcotte et al., 1989; Guiltinan et al., 1990). A homotetramer of a 22-base oligonucleotide that contains either the Em1a or Em1b sequence is sufficient to confer ABA regulation to a minimal -45 cauliflower mosaic virus (CaMV) 35 S core promoter (Marcotte and Quatrano, 1993).

Similar G-box elements have been implicated in responses to diverse environmental signals in plants, including red light (Giuliano et al., 1988), UV light (Weisshaar et al., 1991), and anaerobiosis (McKendree and Ferl, 1992). In addition, a variety of DNA binding proteins that bind the G-box and related sequences have been cloned from plants (reviewed in Katagiri and Chua, 1992). All of the known G-box-specific binding

\footnotetext{
1 Both authors contributed equally to this work.

2 To whom correspondence should be addressed.
}

proteins belong to the basic domain, leucine zipper (bZIP) family of transcription factors. However, with the exception of the genetically defined Opaque2 protein of maize (Schmidt et al., 1990), specific physiological functions have not been assigned to cloned plant bZIP factors. The mechanisms that determine the specificity of G-box-coupled signal transduction pathways in plant cells remain poorly understood. Evidently, the promoter context in which the G-box element is embedded plays an important role. Williams et al. (1992) have shown that sequence differences in the bases immediately flanking the ACGT core affect the protein binding specificity of related elements. In addition, combinatorial interactions between the G-box and other regulatory sequences in the promoter may determine signal specificity (Donald and Cashmore, 1990; Weisshaar et al., 1991; Rogers and Rogers, 1992).

The viviparous1 (vp1) and ABA-insensitive (abi3) mutants identify seed-specific genes required for the ABA response associated with late embryogenesis of maize (McCarty et al., 1989b, 1991) and Arabidopsis (Giraudat et al., 1992), respectively. Analyses of the VP1 protein indicate that it functions as a transcriptional activator (McCarty et al., 1991). Sequence similarities and the analogous phenotypes suggest that $\mathrm{Vp} 1$ and $A b i 3$ are functionally homologous genes (Giraudat et al., 1992). 
Analysis of the maize mutants has shown that both $A B A$ and VP1 are required for induction of the maize $E m$ homolog during embryo development (McCarty et al., 1991). VP1 is also specifically required during seed development for the activation of $\mathrm{C1}$, a regulatory gene of the anthocyanin biosynthetic pathway (McCarty et al., 1989b; Hattori et al., 1992). Our previous studies have shown that overexpression of the VP1 protein in maize cells results in transactivation of chimeric $\beta$-glucuronidase reporter genes (Em-GUS and C1-GUS) in the absence of exogenous ABA. However, several observations suggest that VP1 may interact with these unrelated downstream genes via different mechanisms. First, when ABA is present, the $E m$ and $C 1$ promoters respond differently to VP1 in trans. The Em promoter exhibits a strong positive synergism between VP1 and ABA, whereas the effect on the activation of $C 1$ is less than additive. Second, the colorless/nonviviparous mutant alleles of vp1 differentially affect expression of $C 1$ and the maize Em homolog (McCarty et al., 1989a; McCarty, 1992; Carson, 1993). Third, the cis-acting sequences required for VP1 and ABA regulation of C1 (Hattori et al., 1992) are evidently dissimilar to the G-box $A B A$ response sequences that have been characterized in the wheat $E m$ promoter.

Although the $\mathrm{Cl}$ promoter contains an Em1a-like motif (TACGTGGC), this element is not required for ABA or VP1 regulation. Hattori et al. (1992) showed that the cis elements required for VP1 transactivation and $A B A$ regulation of the $C 1$ promoter are partially separable. A conserved element in the $C 1$ promoter (TCGTCCATGCATGCAC) is essential for both ABA regulation and VP1 transactivation. An adjacent sequence (GTGTCGTG) is specifically required for $A B A$ regulation and not VP1 transactivation.

To better understand the relationship between ABA and VP1 regulatory elements in the $C 1$ and $E m$ promoters and to gain insight into the role of VP1 in ABA signaling, we mapped cisacting sequences in the wheat $E m$ promoter that mediate VP1 transactivation. In contrast with the $\mathrm{C1}$ gene, we show that the complex I G-box elements, Em1a and Em1b, are strongly coupled to VP1 transactivation as well as to ABA regulation of the Em gene. Furthermore, we show that oligonucleotide tetramers of Em1a, Em1b, and a G-box consensus sequence are sufficient for VP1 transactivation and for the interaction with ABA. However, as was found in $C 1$, separable elements in the $E m$ promoter can support VP1 transactivation in the absence of ABA responsiveness.

\section{RESULTS}

\section{The Sequences Necessary for VP1 Transactivation Overlap the ABA Response Elements in the Em Promoter}

To determine whether the cis-acting sequences required for VP1 transactivation colocalized with ABA-responsive elements in the wheat $E m$ promoter, a series of $5^{\prime}$ terminal and internal deletions of the $E m$ promoter were tested for responsiveness to VP1 and ABA by using a maize protoplast transient expression assay (McCarty et al., 1991).

Figure 1 shows that the AT-rich sequences upstream of -243 are not essential for transactivation by VP1 or ABA regulation of $E m$-GUS. This result agrees with the work of Marcotte et al. (1989). Relative to the full-length promoter Em-GUS construct, VP1 transactivation and ABA activation were reduced quantitatively from 30 to $70 \%$ by the deletions of sequences upstream of -180 (BM220.10). Tests of two additional proximal $5^{\prime}$ deletion constructs with break points at -145 (BM220.09)

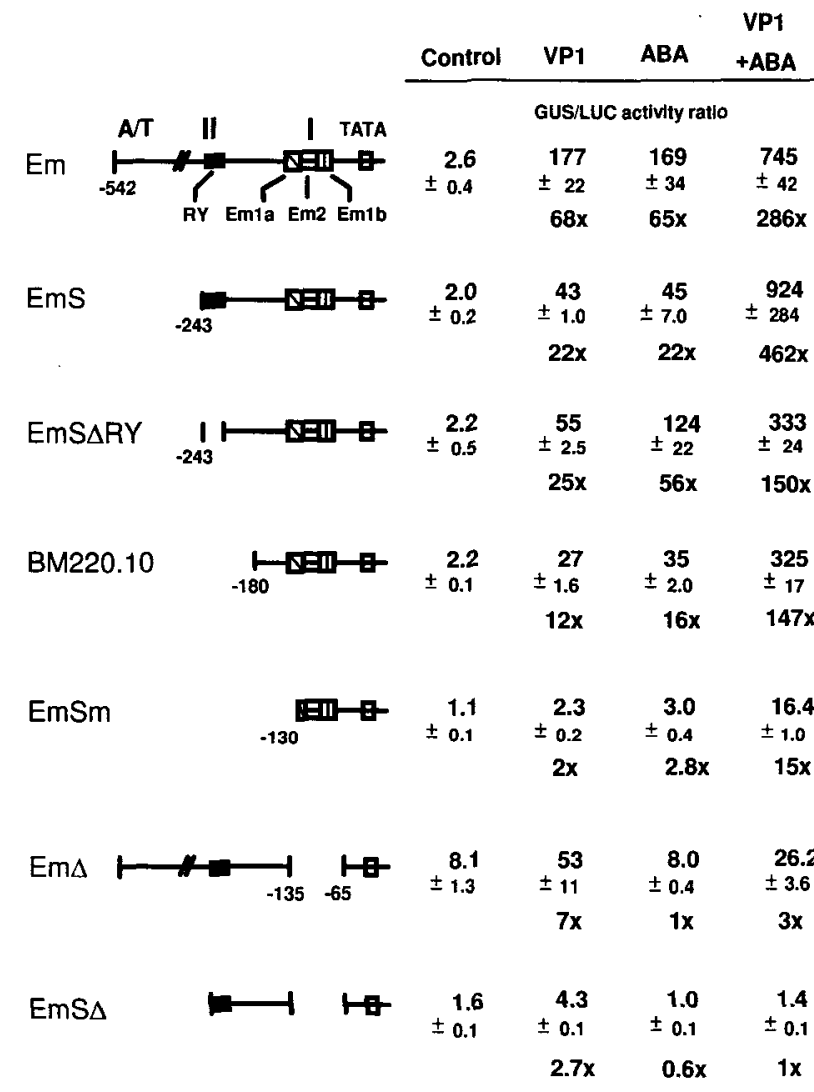

Figure 1. Localization of VP1 Response Sequences in the Em Promoter.

Transactivation of a GUS reporter gene fused to various deletion derivatives of the $E m$ promoter by overexpression of VP1 was determined by cotransfection of 35S-Sh1-VP1 (VP1 treatment) and reporter plasmids into maize protoplasts as described by McCarty et al. (1991). The control and ABA-treated protoplasts received only the GUS reporter gene and the 35S-luciferase control plasmid DNA. The locations of the Em1a, Em2, and Em1b complex (I), RY motif (II), and AT-rich (A/T) upstream sequences are diagrammed for the $E m$ construct. Following electroporation, protoplasts were incubated for $40 \mathrm{hr}$ in media (Vasil et al., 1989) containing no $A B A$ or $10^{-4} \mathrm{M} A B A$. Each value expressed as the relative ratio of GUS-to-luciferase (LUC) activity represents the mean of three independent electroporations with the indicated standard error. The fold activation $(X)$ was determined relative to the corresponding control treatment. 
and -160 (BM220.11) gave results that were similar to BM220.10 (data not shown), indicating that no essential sequences are located upstream of -145 .

Our previous studies of VP1 response sequences in the $C 1$ promoter delineated the Sph regulatory element, which includes an RY sequence motif (Hattori et al., 1992). A 4-bp deletion (EmS $\triangle R Y$ ), which specifically targeted a similar $R Y$ motif located at -220 in the Em promoter, did not significantly affect VP1 activation relative to the EmS construct. Removal of the RY region, however, was correlated with a two- to threefold reduction in the strength of the synergistic interaction of ABA and VP1.

Figure 1 shows that a further truncation of the Em promoter to -130 (construct $\mathrm{EmSm}$ ) severely inhibited responsiveness to VP1 or ABA alone. The deletion break point in the EmSm promoter disrupted the Em1a element, which has been strongly implicated in ABA regulation by the work of Marcotte et al. (1988, 1989) and Guiltinan et al. (1990). This result indicates that Em1a is an essential component of both VP1 transactivation and ABA regulation. The EmSm construct, however, was still activated significantly and exhibited a synergistic response to VP1 and $A B A$ in combination, suggesting that a second $G$-box motif in complex I, Em1b, could play a role as suggested by previous studies of the Em promoter (Marcotte et al., 1989; Marcotte and Quatrano, 1993).

To test the involvement of this complex, a 70-bp internal deletion (bases - 65 to -135) that removed both Em1a and Em1b from the promoter was constructed. Figure 1 shows that this deletion (construct $\mathrm{Em} \Delta$ ) abolished ABA activation. In contrast, Em $\Delta$ remained significantly responsive to VP1 transactivation (sevenfold over the basal activity). Although the Em $\Delta$ promoter remained responsive to VP1 in trans, the synergistic interaction with ABA was lost. Instead, VP1 transactivation of this promoter was inhibited slightly by the addition of hormone. Truncation of this mutant promoter to $-243(\mathrm{EmS} \Delta)$ strongly inhibited transactivation by VP1, indicating that the principal elements responsible for hormone-independent transactivation are located upstream of position -243. Weak, residual VP1 activation (less than threefold) of the EmS $\Delta$ construct, which retained the RY domain, was detected in two of four independent experiments (data not shown). Given the effects of the RY deletion shown above, these data do not support the integral involvement of the RY motif in hormone-independent VPI transactivation.

\section{Complex 1 Is Sufficient To Confer VP1 Transactivation to a Viral Promoter}

Guiltinan et al. (1991) have shown that a 76-bp fragment containing complex $I$ is sufficient to confer $A B A$ regualtion to the -90 core region of the CaMV $35 S$ promoter (BM226.5). Figure 2 shows that this construct is transactivated by VP1 in maize cells. The BM233.3 construct, which contains a two-base mutation in the Em1a sequence, was less strongly activated by VP1. Consistent with the promoter deletion analysis, mutagenesis

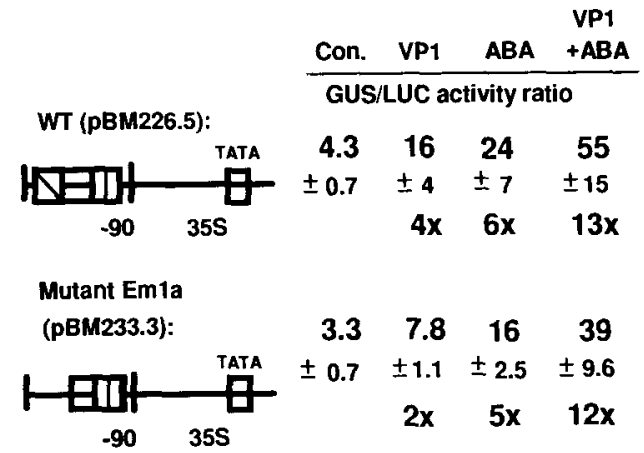

Figure 2. Complex I Is Sufficient for VP1 Transactivation and the Synergistic Interaction with $\mathrm{ABA}$.

BM226.5, which contains a 76-bp promoter fragment that includes the Em1a and Em1b elements fused to the -90 core region of the CaMV $35 \mathrm{~S}$ promoter (Guiltinan et al., 1990), was tested for responsiveness to VP1 and ABA in the protoplast transactivation assay described in Figure 1. The BM233.3 construct contains a two-base change mutation in the Em1a element (CACGTGGC $\rightarrow$ CCCGgGGC where lowercase letters indicate mutated bases). VP1 and ABA activations were determined, as described in Figure 1 , with $10^{-4} \mathrm{M}$ ABA. The boxes representing promoter elements and VP1 and ABA activations are as given in Figure 1. Con., control; LUC, luciferase; WT, wild type; 35S, -90 core component of the CaMV 355 promoter.

of Em1a alone did not completely block VP1 and ABA regulation of this construct.

\section{Multimerized G-Box Family Sequences Are Sufficient To Mediate VP1 and ABA Regulation and Their Synergistic Interaction}

To resolve further the minimum sequence needed to confer VP1 and ABA regulation in the context of a minimal promoter, 22-mer oligonucleotide sequences were tetramerized and cloned upstream of the -45 core TATA region of the CaMV $35 S$ promoter fused to the GUS gene. Figure 3 shows that tetramer promoters, which included either Em1a or Em1b with their immediate flanking sequences, reproduced the essential features of VP1 and ABA regulation observed with the native Em promoter. In combination, VP1 and ABA were strongly synergistic and resulted in 33- and 118-fold activation of the Em1a and Emib tetramer constructs, respectively. Although Em1a and Em1b are flanked by apparently dissimilar sequences, the importance of context cannot be ruled out completely. To address this possibility, a tetramer of a 24-bp sequence containing the consensus dyad G-box element from the parsley chalcone synthase gene (CHS) promoter (Weisshaar et al., 1991) was tested. As shown in Figure 3, this promoter produced a response that was comparable to the Em1a miminal promoter construct. A four-base mutant derivative of the CHS G-box tetramer was completely unresponsive to VP1 and ABA, 


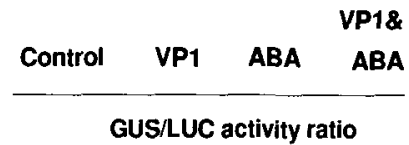

Em1a 22-mer:

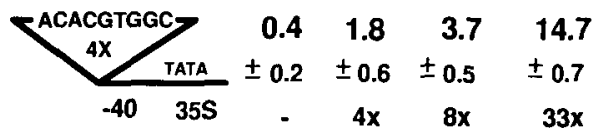

Em1b 22-mer:

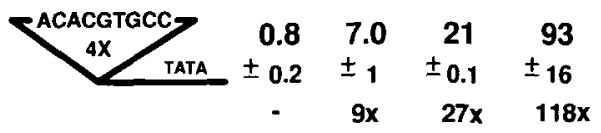

G-box:

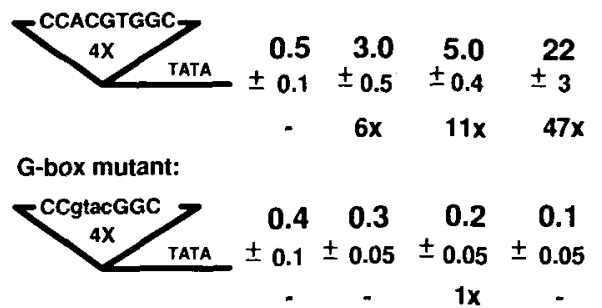

Figure 3. Multimerized G-Box Elements Confer VP1 and ABA Responsiveness, and VP1 and ABA Synergism, to a Minimal Promoter.

Synthetic 22-bp oligonucleotides that contained the Em1a or Em1b sequences were cloned four times as tetramers upstream of the -45 CaMV $35 S$ core promoter sequence fused to the GUS gene (Marcotte and Quatrano, 1993). A tetramer of a G-box element with dyad symmetry was derived from a 24-bp oligonucleotide sequence that included box I of the UV light-regulated CHS promoter from parsley (Weisshaar et al., 1991). The mutant G-box tetramer construct was identical in sequence except for the four-base substitution in each copy of the ACGT core as indicated. VP1 and ABA activations were determined, as described in Figure 1, with $10^{-4} \mathrm{M}$ ABA. Dashes indicate fold activations equal to or less than one. LUC, luciferase.

confirming that the ACGT core motifs of the oligonucleotide inserts were necessary for the response.

\section{The Em1a and Em1b Elements Are Not Functionally Redundant in Their Native Context}

The experiments in Figure 3 indicate that either Em1a or Em1b can mediate VP1 activation in the context of a homotetramer promoter. To determine whether one or both elements are functionally important in their normal context in the native $E m$ promoter, two-base mutations were introduced into the Em1a and Em1b elements of the EmS construct by oligonucleotidedirected mutagenesis. Figure 4 shows that two-base mutations converting the ACGT cores of either Em1a or Em1b to CCGG (Emx1a and Emx1b, respectively) strongly inhibited $A B A$ induction. The Em1b mutant inhibited $A B A$ regulation but not VP1 transactivation, suggesting that these elements have differential roles in Em regulation. However, both Em1a and Em1b mutant constructs were synergistically activated by VP1 and $A B A$ in combination. The Em1a and Em1b mutants caused similar inhibition of $A B A$ and mannitol activation of the $E m$ promoter in rice protoplasts (J.L. Magnard and R.S. Quatrano, unpublished results).

\section{DISCUSSION}

Our data delineate and compare the cis-acting sequences in the Em promoter required for VP1 transactivation and $\mathrm{ABA}$ regulation, respectively. These results led us to three conclusions. First, G-box elements are sufficient to mediate VP1 transactivation as well as the synergistic interaction of VP1 and ABA observed in maize cells. Second, separable elements located upstream of the G-box complex in the wheat $E m$ promoter confer VP1 responsiveness without supporting ABA regulation or the synergistic VP1 and ABA interaction. Third, in contrast with its essential function in the $C 1$ promoter, the $R Y$ sequence motif does not play a major role in VP1 or ABA regulation of $E m$ in protoplasts.

\section{The Sufficiency and Specificity of G-Box Elements}

Closely related G-box regulatory sequences have been implicated in a variety of signaling pathways in plants, including

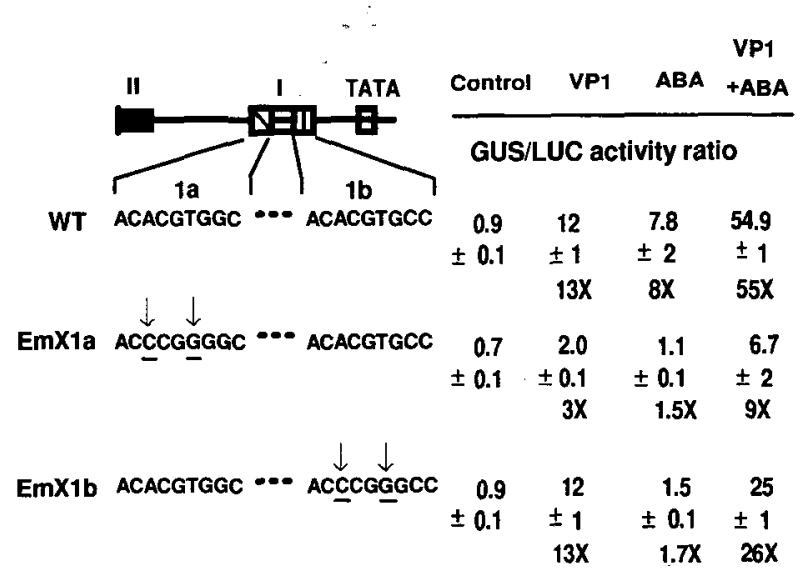

Figure 4. Directed Mutatgenesis of the Em1a and Em1b Elements in Their Normal Promoter Context.

Two base change substitutions were made in the Em1a and Em1b motifs, respectively, and in the EmS promoter by oligonucleotide-mediated directed mutagenesis (see Methods). Electroporations and GUS activation assays were performed as described in the legend to Figure 1 , using $10^{-5} \mathrm{M} A B A$ treatments. Mutated bases are indicated with downward arrows and are underlined. The dashes indicate sequences between Em1a and Em1b that are not shown. Abbreviations are as given in Figure 1. Emx1a, 2-bp mutant in the Emia element; Emx1b, 2-bp mutant in Em1b; WT, wild type. 
red light (Donald and Cashmore, 1990), UV light (Weisshaar et al., 1991), anaerobiosis (McKendree and Ferl, 1992), and ABA (Marcotte et al., 1989). Two mechanisms believed to contribute to the specificity of the responses mediated by the G-box motif are (1) differences in the sequences flanking the ACGT core that determine affinity for DNA binding factors (Williams et al., 1992) and (2) combinatorial interactions between G-box binding factors and other regulatory elements in the promoters (Donald and Cashmore, 1990; Weisshaar et al., 1991). The latter studies indicate that the G-box element alone is not sufficient to confer light activation. In contrast, studies in several systems have shown that a multimerized (four to six tandem copies) G-box or closely related motif is sufficient to confer to ABA responsivity to a minimal promoter in plant cells (Lam and Chua, 1991; Skriver et al., 1991; Marcotte and Quatrano, 1993). Our results showed that as tetramers, any of several G-box-like sequences, including the parsley CHS G-box normally associated with light regulation, can confer $A B A$ as well as VP1 responsiveness in maize protoplasts. The fact that the Em1a, Em1b, and CHS G-box oligonucleotides tested contained dissimilar flanking sequences implies that the core G-box motif by itself confers the necessary specificity for VP1 transactivation.

At a minimum, the tetramer experiments identified a context in which the G-box is sufficient for ABA and VP1 regulation; however, extrapolation of this result to the function of the Em1a and Em1b elements in their normal context should be tempered by at least two considerations.

First, the consequences of multimerizing the G-box element are poorly understood. Multimerization could introduce cooperative interactions or other nonphysiological effects that would mask a requirement for interactions with other components in the natural context. We have noted that as a monomer, the G-box is not sufficient for ABA regulation in rice protoplasts (Marcotte and Quatrano, 1993; W.R. Marcotte, unpublished results). A situation in which multiple copies of a single component can substitute where a complex of heterologous elements is required in the normal promoter has been described for the gibberellin response complex in an $\alpha$-amylase promoter of barley (Rogers and Rogers, 1992). Remarkably, Rogers and Rogers (1992) showed that in the same system, a single Em1a element could confer ABA responsiveness when combined with heterologous components of the gibberellin response complex. In yet another context, we have found that the solitary Em1a-like element (tACGTGGC) located at a comparable position in the C1 promtoer is essential for light regulation of that gene (C.-Y. Kao, I.K. Vasil, and D. R. McCarty, unpublished results) but does not function in ABA signaling or VP1 transactivation (Hattori et al., 1992). Overall, these observations are consistent with the idea that in a normal context, the functional specificity of the G-box is conditioned by interactions with other regulatory elements, as has been proposed for other signaling pathways. VP1-coupled elements in the $E m$ promoter upstream of position -243 and elements such as Em2 (J.L. Magnard and R.S. Quatrano, unpublished results) could play a role in this type of coupling interaction.
Second, the tetramer results do not imply that Em1a and Em1b necessarily have equivalent functions. The mutagenesis experiments indicated that in their normal context, these elements are not simply redundant. In their native context, both Em1a and Em1b participate in ABA regulation but contribute differentially to VP1 transactivation. Moreover, in vitro footprinting studies indicate that Em1a and Em1b have different specificities for DNA binding proteins (Guiltinan et al., 1990), suggesting that they may bind different factors in vivo. One possibility is that there is sufficient overlap in the specificity of Em1a and Em1b binding factors to allow a homotetrameric site to substitute for a heteromeric Em1a-Em1b complex.

\section{The Role of VP1 in Gene Activation and ABA Signaling}

We have shown that the $A B A$ response complex in Em also mediates VP1 transactivation and ABA and VP1 synergism. The evidence that $A B A$ induction of $E m$ does not require protein synthesis (Williamson and Quatrano, 1988) suggests that the VP1 activator acts directly through the G-box elements rather than through the activation of intermediate regulatory genes. Although VP1 has a functionally defined transcriptional activation domain, there is little evidence that VP1 by itself binds directly to DNA (McCarty et al., 1991). Although we speculate that VP1 may function as a coactivator that interacts with the G-box via protein-protein contacts with G-box-specific DNA binding proteins, direct evidence of physical interactions with known G-box binding proteins is currently lacking. The recombinant VP1 protein has not been shown to interact detectably with G-box oligonucleotide probes in mobility shift assays, and VP1 antibodies failed to perturb G-box binding activities present in embryo extracts (B. Li and D.R. McCarty, unpublished results). Until the native biochemical properties of the VP1 protein and G-box binding protein complexes are better understood, it is difficult to interpret the negative results of the in vitro studies.

The observation that VP1 can also activate Em transcription through cis elements that are separable from the ABA response complex suggests that VP1 can interact with a second class of regulatory elements. A similar distinction between VP1- and ABA-responsive sequences has been demonstrated in the C1 promoter (Hattori et al., 1992). In the C1 promoter, the Sph element (TCGTCCATGCATGCAC) is necessary (Hattori et al., 1992) and sufficient for VP1 regulation (C.-Y. Kao, I.K. Vasil, and D.R. McCarty, unpublished results). The 3 portion of the Sph domain of $C 1$ contains the RY sequence motif (Dickinson et al., 1988). Our data, however, indicate that a similar RY motif located in the proximal region of the Em promoter has at most minor involvement in VP1 and ABA regulation and is not sufficient for VP1 transactivation. These results are consistent with evidence that the functionally defined boundaries of Sph extend beyond the RY sequence motif in C1 (Hattori et al., 1992; C.-Y. Kao, I.K. Vasil, and D.R. McCarty, unpublished results). On the other hand, upstream elements located 
between positions -243 and -542 in conjunction with the core TATA region of the wheat $E m$ promoter are sufficient for hormone-independent transactivation of $E m$. Although this ATrich upstream region lacks good G-box consensus motifs, it includes a second RY motif at position -389 (TGCATGCAT). The sequences flanking this RY, however, do not otherwise closely resemble the Sph domain of $\mathrm{C1}$.

Our data indicate that the synergistic interaction between VP1 and ABA is a characteristic of the G-box-mediated response that does not require the upstream VP1 response sequences. This is consistent with the finding that G-box-type elements are not involved in the nonsynergistic VP1-ABA response mediated by the GTGTC-Sph complex of C1 (Hattori et al., 1992). The fact that both the Emx1a and Emxlb mutants were synergistically activated by VP1 and ABA (Figure 4) indicates that multiple G-box copies are not required for synergism. This result does not support models that invoke cooperative DNA binding of G-box binding factors (GBFs) as the sole basis for synergism. Another possibility is that two classes of GBF proteins occupy these sites: a constitutive class that has a lowbinding affinity for VP1, and an ABA-induced class that has a high-binding affinity for VP1 and is capable of causing weak activation in the absence of VP1. In this scenario, VP1 could transactivate $E m$ in the absence of a hormone via the weak interaction with the constitutive GBF. In the presence of $A B A$, VP1 would activate more strongly because the GBF with low VP1 affinity would be replaced by the GBF with high VP1 affinity.

A superficial similarity in the organization of conserved elements in the two promoters shown in Figure 5 belies the evidence that different combinations of elements mediate $A B A$ and VP1 regulation of the $C 1$ and $E m$ genes. A capacity to interact with factors that bind the ABA-specific sites as well as hormone-independent regulatory sequences might allow the VP1 activator to participate in a coupling or integration of the $A B A$ response with other intrinsic developmental signals (Hattori et al., 1992; Rogers and Rogers, 1992; McCarty, 1993). The RY motif (CATGCATG), which may be at least one component of Sph, has been implicated in seed-specific gene expression in legumes (Dickinson et al., 1988; Baumlein et al., 1992). The divergence of the Sph-RY region in the promoter of the $p /$ gene, which is the otherwise highly conserved non-seed tissue-specific homolog of $\mathrm{C} 1$ in maize (Cone et al., 1993), is consistent with this hypothesis. The relevance of $R Y$ motifs to developmental regulation of the Em gene remains unclear. That Em regulation is evidently not strongly coupled to $R Y$ is nevertheless consistent with the observation that unlike $C 1$ (Cone et al., 1993), Em expression is not strictly limited to seed tissues (Berge et al., 1989).

\section{Differential Regulation of the $E m$ and $C 1$ Genes}

The finding that VP1 activation is mediated by dissimilar sequences in the $E m$ and $C 1$ promoters suggests a basis for the differential regulation of the corresponding genes by $A B A$ and VP1 in maize. Because of the synergism afforded by the G-box
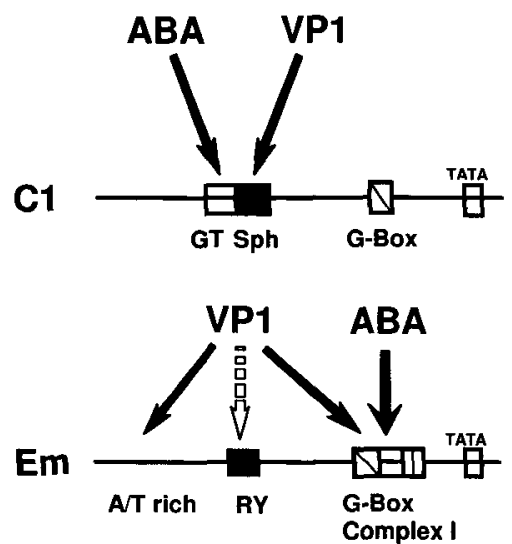

Figure 5. Regulatory Components of the Em and C1 Promoters involved in VP1 and ABA Regualtion.

The key regulatory elements involved in VP1 transactivation and ABA regulation of the $E m$ and $C 1$ genes, respectively, are summarized schematically. The scheme for $C 1$ is derived from Hattori et al. (1992). In both promoters, an $A B A$ response sequence occurs in conjunction with a separable element capable of mediating VP1 transactivation alone. In $\mathrm{C1}$, hormone-independent transactivation is mediated by the Sph element, whereas a similar RY motif present in Em is not essential for VP1 or ABA regulation. Hormone-independent activation of $E m$ by VP1 is mediated by promoter elements located between positions -243 and -542 . GT is a GTGTCGTGTC sequence motif adjacent to $\mathrm{Sph}$ that is required for $A B A$ regulation of $C 1$. The composition of G-box complex $I$ is given in the legend to Figure 1. The filled boxes represent the RY sequence motif present in the Em promoter and is one component of the Sph regulatory element.

complex, the wheat $E m$ promoter has greater responsiveness to ABA. Quantitatively, Em expression is more sensitive to changes in hormone level. This might account for the observation that expression of the maize $E m$ homolog is greatly reduced in ABA-deficient mutants (McCarty et al., 1991), although such mutants do not block anthocyanin expression in the seed. Analysis of anthocyanin-deficient, nonviviparous vp1 alleles, which differentially affect regulation of maize $\mathrm{Em}$ and C1 genes in the developing seed (McCarty et al., 1989a; McCarty and Carson, 1990; McCarty, 1992; Carson, 1993), and mutagenesis studies of the VP1 protein (L. Rosenkrans, S. Cocciolone, and D.R. McCarty, manuscript in preparation) suggest that activation of the two genes involves different functional domains in the VP1 protein. Whether the wheat $E m$ and maize C1 promoters typify broader classes of ABA-regulated genes or whether still other motifs are employed remains to be determined.

\section{METHODS}

\section{Protoplast Transient Expression Assays}

Protoplasts were prepared from maize suspension culture cells for electroporation, as described previously (Vasil et al., 1989; McCarty 
et al., 1991). Protoplast samples $\left(4 \times 10^{6}\right.$ cells) were electroporated with $10 \mathrm{mg}$ of the $\beta$-glucuronidase (GUS) plasmid DNA, $10 \mu \mathrm{g}$ of a ubiquitin promoter-luciferase plasmid included as an internal standard (Christensen et al., 1992), and as indicated, $10 \mu \mathrm{g}$ of the cauliflower mosaic virus (CaMV) 35S-Sh1-VP1 effector plasmid described in McCarty et al. (1991). Following electroporation, each sample was divided into equal subsamples, and they were incubated for $48 \mathrm{hr}$ in media that contained no abscisic acid (ABA) or hormone $\left(10^{-4}\right.$ or $10^{-5} \mathrm{M} \mathrm{ABA}$ as indicated). GUS assays were performed using the method of Jefferson (1987), with modifications described in Rosenkrans et al. (1995). The luciferase assays, modified from the method of Millar et al. (1992), were performed by mixing $10 \mu \mathrm{L}$ of extract and $200 \mu \mathrm{L}$ of reaction buffer $\left(25 \mathrm{mM}\right.$ Tricine, $\mathrm{pH} 7.8,15 \mathrm{mM} \mathrm{MgCl}_{2}, 5 \mathrm{mM}$ ATP $0.05 \%[\mathrm{~W} / \mathrm{v}] \mathrm{BSA}$ ) in the cuvette of a Monolight 2010 luminometer (Analytical Luminescence Laboratory, San Diego, CA). The reactions were initiated automatically in the luminometer by injection of $100 \mu \mathrm{L}$ of $1 \mathrm{mM}$ luciferin, and the emitted photons were counted for a period of $15 \mathrm{sec}$. The GUS activities reported were normalized to the luciferase activity.

\section{Plasmid Constructions}

The construction of the pBM113Kp (wild-type EM-GUS) and BM220.10 plasmids is described in Marcotte et al. (1988, 1989). EmS was constructed by digestion of pBM113Kp DNA with Sall and religation of the backbone fragment. To construct Em $\times 1 a$ and $E m \times 1 b$, two base change mutations were introduced into the respective Em1a and Emtb ACGT motifs in the EmS promoter by oligonucleotide-directed mutagenesis performed using the Altered Sites system according to manufacture's instructions (Promega). For mutagenesis, the Ems gene was subcloned into the pSelect plasmid (Promega). The mutant oligonucleotides 5'-GCTGTCGCGCCCCGGGTCCGGCAAGG-3' (Em1a specific) and 5'-GAGGCGGCCCGGGTGCAGCG-3' (Em1b specific) created Smal sites at Em1a and Em1b, respectively. The mutants were confirmed by DNA sequencing and subcloned back into pUC19 for transient expression analysis. The EmSm promoter deletion was constructed by digestion of Emxla with Smal and religation of the backbone fragment.

The $\mathrm{Em} \Delta$ internal deletion was constructed by first generating a pair of Nael restriction sites in the BM113Kp promoter by oligonucleotidedirected mutagenesis as described earlier. The mutagenic oligonucleotides 5'-CGCCACTGGCCGGCAAG-3' and 5'-GTGAAGCACGCCGGCGGCACGT $-3^{\prime}$ were designed to generate $\mathrm{Nael}$ sites at positions -65 and -135 , and were used simultaneously to prime second strand DNA synthesis in the Altered Sites system to generate both sites in a single step. The doubly mutated sequence was subcloned back into pUC19, and the resulting plasmid was digested with Nael. The large Nael backbone fragment was recircularized to generate EM $\Delta$. The deletion was confirmed by DNA sequencing. $\mathrm{EmS} \Delta$ was derived from $\mathrm{Em} \Delta$ by digesting the former plasmid with Sall and religating the backbone fragment.

To construct EmS $\triangle R Y$, a directed three-base mutation was made in the EmS promoter using an oligonucleotide, 5'-GACTCTGCTTGGATCCATGGATTGTCGACG-3', that created a BamHI and a Ncol restriction site in the Sph element. The mutant plasmid was digested with $\mathrm{Ncol}$, treated with $\mathrm{S} 1$ nuclease to remove the four-base overhand, and religated.

The Em1a and Em1b tetramer plasmids are described by Marcotte and Quatrano (1993). The chalcones synthase (CHS) G-box tetramer construct is described in Weisshaar et al. (1991). The pBM226.5 and pBM233.3 plasmids are described in Guiltinan et al. (1990).

\section{ACKNOWLEDGMENTS}

We thank Margaret Stoll for technical assistance, Berndt Weisshaar for the CHS tetramer constructs, and Chien-Yuan Kao and Christian B. Carson for helpful discussions. This work was supported by grants from the National Science Foundation to D.R.M. (Nos. DCB91032279 and IBM 9406692) and from the National Institutes of Health to R.S.Q. (No. GM44288). This paper is Florida Agricultural Experiment Station Journal Series number R-04583.

Received April 10, 1995; accepted July 5, 1995.

\section{REFERENCES}

Baumlein, H., Nagy, I., Villarroel, R., Inzé, D., and Wobus, U. (1992). cis analysis of a seed protein gene promoter: The conservative RY repeat CATGCATG within the legumin box is essential for tissuespecific expression of a legumin gene. Plant J. 2, 233-239.

Berge, S.K., Bartholomew, D.M., and Quatrano, R.S. (1989). Control of the expression of wheat embryo genes by abscisic acid. In Molecular Basis of Plant Development, R.B. Goldberg, ed (New York: Alan R. Liss), pp. 193-201.

Carson, C.B. (1993). Characterization of the Structure and Expression of the Mutant Alleles of the viviparous-1 Gene of Maize. PhD Dissertation (Gainesville, FL: University of Florida).

Christensen, A.H., Sharrock, R.A., and Quail, P.H. (1992). Maize ubiquitin genes: Structure, thermal perturbation of expression and transcript splicing, and promoter activity following transfer to protoplasts by electroporation. Plant Mol. Biol. 18, 675-689.

Cone, K.C., Cocciolone, S.M., Moehlenkamp, C.A., Weber, T., Drummond, B.J., Tagliani, L.A., Bowen, B.A., and Perrot, G.H. (1993). Role of the regulatory gene $p /$ in the photocontrol of maize anthocyanin pigmentation. Plant Cell 5, 1807-1816.

Dickinson, C.D., Evans, R.P., and Neilsen, N.C. (1988). RY repeats are conserved in the 5 '-flanking regions of legume seed-protein genes. Nucleic Acids Res. 16, 371.

Donald, R.G.K., and Cashmore, A.R. (1990). Mutation of either G-box or $\mathrm{l}$-box sequences profoundly affects expression from the Arabidopsis rbcs-1A promoter. EMBO J. 9, 1717-1726.

Giraudat, J., Hauge, B.M., Valon, C., Smalle, J., Parcy, F., and Goodman, H.M. (1992). Isolation of the Arabidopsis $A B / 3$ gene by positional cloning. Plant Cell 4, 1251-1261.

Giuliano, G., Pichersky, E., Malik, V.S., Timko, M.P., Scolnik, P.A., and Cashmore, A.R. (1988). An evolutionary conserved protein binding sequence upstream of a plant light-regulated gene. Proc. Natl. Acad. Sci. USA 85, 7089-7093.

Guiltinan, M.J., Marcotte, W.M., and Quatrano, R.S. (1990). A leucine zipper protein that recognizes an abscisic acid response element. Science 250, 267-271.

Hattori, T., Vasil, V., Rosenkrans, L., Hannah, L.C., McCarty, D.R., and Vasil, I.K. (1992). The viviparous-1 gene and abscisic acid activate the $C 1$ regulatory gene for anthocyanin biosynthesis during seed maturation in maize. Genes Dev. 6, 609-618.

Jefferson, R.A. (1987). Assaying chimeric genes in plants: The GUS gene fusion system. Plant Mol. Biol. Rep. 3, 387-405. 
Katagiri, F., and Chua, N.-H. (1992). Plant transcription factors: Present knowledge and future challenges. Trends Biochem. Sci. 8, 2-27.

Lam, E., and Chua, N.-H. (1991). Tetramer of a 21-base pair synthetic element confers seed expression and transcriptional enhancement in response to water stress and abscisic acid. J. Biol. Chem. 266, 17131-17135.

Marcotte, W.R., Jr., and Quatrano, R.S. (1993). ABA-regulated gene expression: cis-acting sequences. In Plant Responses to Cellular Dehydration during Environmental Stress, T.J. Close and E.A. Bray, eds (Rockville, MD: American Society of Plant Physiologists), pp. 185-192.

Marcotte, W.R., Jr., Bayley, C.C., and Quatrano, R.S. (1988). Regulation of a wheat promoter by abscisic acid in rice protoplasts. Nature 335, 454-457.

Marcotte, W.R., Jr., Russell, S.H., and Quatrano, R.S. (1989). Abscisic acid-responsive sequences from the Em gene of wheat. Plant Cell 1, 969-976.

McCarty, D.R. (1992). The role of VP1 in regulation of seed maturation in maize. Biochem. Soc. Trans. 20, 89-92.

McCarty, D.R. (1993). The role of the maize viviparous-1 gene in regulation of seed maturation. In Cellular Communication in Plants, R.M. Amasino, ed (New York: Plenum Press), pp. 27-36.

McCarty, D.R., and Carson, C.B. (1990). Molecular genetics of seed maturation in maize. Plant Physiol. 81, 267-272.

McCarty, D.R., Carson, C.B., Lazar, M., and Simonds, S.C. (1989a). Transposable element induced mutations of the viviparous-1 gene of maize. Dev. Genet. 10, 273-281.

McCarty, D.R., Carson, C.B., Stinard, P.S., and Robertson, D.S. (1989b). Molecular analysis of viviparous-1: An abscisic acidinsensitive mutant of maize. Plant Cell 1, 523-532.

McCarty, D.R., Hattori, T., Carson, C.B., Vasil, V., Lazar, M., and Vasil, I.K. (1991). The viviparous-1 developmental gene of maize encodes a novel transcriptional activator. Cell 66, 895-905.

McKendree, W.L., and FerI, R.J. (1992). Functional elements of the Arabidopsis Adh promoter include the G-box. Plant Mol. Biol. 19, 859-862.

Millar, A.J., Short, S.R., Hiratsuka, K., Chua, N.-H., and Kay, S.A. (1992). Firefly luciferase as a reporter of regulated gene expression in higher plants. Plant Mol. Biol. Rep. 10, 324-337.
Quatrano, R.S., Guiltinan, M.J., and Marcotte, W.R., Jr. (1993). Regulation of gene expression by abscisic acid. In Control of Plant Gene Expression, D.P.S. Verma, ed (Boca Raton, FL: CRC Press), pp. 69-90.

Rock, C., and Quatrano, R.S. (1994). The role of phytohormone during seed development. In Plant Hormones and Their Roles in Plant Growth and Development, P.J. Davies, ed (Boston: Martinus Nijhoff), pp. 671-697.

Rogers, J.C., and Rogers, S.W. (1992). Definition and functional implications of gibberellin and abscisic acid cis-acting hormone response complexes. Plant Cell 4, 1443-1451.

Rosenkrans, L., Vasil, V., Vasil, I.K., and McCarty, D.R. (1995). Functional analysis of a plant transcription factor using transient expression in maize protoplasts. In Methods in Plant Molecular Biology, P. Maliga, D.F. Klessig, A.R. Cashmore, W. Gruissem, and J.E. Varner, eds (Cold Spring Harbor, NY: Cold Spring Harbor Laboratory Press), pp. 19-36.

Schmidt, R.J., Burr, F.A., Aukerman, M.J., and Burr, B. (1990). Maize regulatory gene Opaque-2 encodes a protein with a "leucine-zipper" motif that binds to zein DNA. Proc. Natl. Acad. Sci. USA 87, 46-50.

Skriver, K., and Mundy, J. (1990). Gene expression in response to abscisic acid and osmotic stress. Plant Cell 2, 503-512.

Skriver, K., Olsen, F.L., Rogers, J.C., and Mundy, J. (1991). cis-acting DNA elements responsive to gibberellin and its antagonist abscisic acid. Proc. Natl. Acad. Sci. USA 88, 7266-7270.

Vasil, V., Clancy, M., Ferl, R.J., Vasil, I.K., and Hannah, L.C. (1989). Increased gene expression by the first intron of the maize sh1 locus in grass species. Plant Physiol. 91, 1575-1579.

Weisshaar, B., Armstrong, G.A., Block, A., da Costa e Silva, O., and Halbrock, K. (1991). Light-inducible and constitutively expressed DNA-binding proteins recognizing a plant promoter element with functional relevance in light responsiveness. EMBO J. 10, 1777-1786.

Williams, M.E., Foster, R., and Chua, N.-H. (1992). Sequences flanking the hexameric G-box core CACGTG affect the specificity of protein binding. Plant Cell 4, 485-496.

Williamson, J.D., and Quatrano, R.S. (1988). ABA-regulation of two classes of embryo-specific sequences in mature wheat embryos. Plant Physiol. 86, 208-215. 